\title{
Trophic Effects on the Contractile and Histochemical Properties of Rat Soleus Muscle ${ }^{1}$
}

\author{
SIDNEY A. SPECTOR ${ }^{2}$ \\ Department of Kinesiology, University of California, Los Angeles, California 90024
}

\begin{abstract}
Contractile and histochemical properties of rat soleus muscle were studied bilaterally after 2 or 4 weeks of denervation (DEN), which eliminates activity and non-activity-related influences, and chronic application of tetrodotoxin (TTX) to the motor nerve, which produces a completely inactive innervated muscle. After 2 or 4 weeks of disuse, the percentages of slow twitch oxidative fibers in both DEN- and TTX-treated soleus were reduced significantly and to similar extents. The dynamic contractile properties of TTX-treated and denervated muscles were similar to those of control muscle after 2 weeks, but by 4 weeks, parallel increases were seen in normalized rate of tension development and maximal shortening speed of these muscles. After either period of disuse, the atrophy of TTX-treated soleus was significantly less than that of denervated muscle. Atrophy was associated with correspondingly diminished capacity of denervated or TTX-disused muscles to generate tension after 2 weeks. By 4 weeks of treatment, the reduction in tension of denervated soleus was greater than its diminished size. These results imply the existence of a non-activity-related stimulus in TTXinactivated muscles which slows the reductions in muscle mass and specific tension observed in denervated soleus muscle. In contrast, the similarities in dynamic speed-related properties and fiber type profiles between DEN- and TTXtreated rat soleus may be explained simply by the absence of neuromuscular activity.
\end{abstract}

The cross-reinnervation experiments of Buller et al. (1960) and others (Buller et al., 1969; Close, 1969; Bagust et al., 1981; Chan et al., 1982) demonstrated that the motoneuron regulates a variety of contractile, biochemical, and histochemical properties of skeletal muscle. The signals responsible for this muscular plasticity were hypothesized to derive from newly directed activity patterns and/or the supply of a neurotrophic substance to the muscle by the innervating nerve (Buller et al., 1960).

Chronic electrical stimulation of innervated or denervated fast twitch muscle at frequencies thought to normally excite slow twitch

Received November 16, 1984; Revised February 27, 1985;

Accepted March 13, 1985

${ }^{1}$ This work was supported by National Institutes of Health Grant NS16333 awarded to $V$. Reggie Edgerton, and was undertaken, in part, at the Jerry Lewis Neuromuscular Research Center. I thank Drs. V. Reggie Edgerton, Earl Homsher, and Michael S. Letinksy for reviewing critically this manuscript. The technical contributions of Nicholas V. Ricchiuti, Cesar Blanco, and Mary C. Carter are appreciated.

${ }^{2}$ Present address: Laboratory of Neural Control, NINCDS, National Institutes of Health, Building 36, Room 5A29, Bethesda, MD 20205. muscle produces striking conversions of the muscle's contractile (Lømo et al., 1974; Salmons and Sreter, 1976; Buller and Pope, 1977), biochemical (Pette et al., 1973; Sreter et al., 1973), histochemical (Pette et al., 1975), and ultrastructural (Eisenberg and Salmons, 1981) characteristics toward those observed normally in slow twitch muscle. Fast-to-slow-type transformations occur after chronic stimulation of fast twitch muscle at high frequencies, as well (Sreter et al., 1982). Thus, the phenotypic expression of muscle appears to depend to a significant degree on one or more characteristics related to neuromuscular activity.

Few studies, however, have attempted to assess the role of trophism in controlling the contractile and histochemical properties of muscle. Blockade of axoplasmic transport with cholchicine causes a modest slowing of twitch contraction and relaxation times of rat muscle (Hofmann and Peacock, 1973), although this effect has not been confirmed (Fernandez and Ramirez, 1974). Experiments in which chronic treatment with botulinum toxin (which blocks presynaptic release of vesicular acetylcholine; Kao et al., 1976) produces contractile behavior similar to that seen in contralaterally denervated muscle (Drachman and Johnston, 1975; Brown et al., 1982) suggest that cholinergic transmission, in the form of cvokcd or spontaneous vesicular emission, is responsible for regulation of these properties. Nevertheless, the possibility that botulinum toxin prevents the release of an additional putative trophic factor in association with, or independent of, release of vesicular acetylcholine cannot be excluded. In addition, these studies do not distinguish between effects produced by elimination of spontaneously released factors as opposed to the absence of nerve-evoked activity, as both of these components are removed with botulinum treatment.

Therefore, the present experiment was designed to differentiate, by means of pharmacological nerve block, a potential trophic stimulus from neuromuscular activity in regulating contractile function of muscle. Comparisons were made between the effects of 2 or 4 weeks of denervation (DEN) of the rat soleus, which eliminates both trophic and activity-related influences, and the changes seen after chronic application to the motor nerve of tetrodotoxin (TTX), which eliminates neuromuscular activity while permitting normal axonal transport and synaptic transmission (Pestronk et al., 1976; Lavoie et al., 1977; Bray et al., 1979). Bilateral comparisons of these treatments were performed with respect to isometric and dynamic contractile properties and histochemical fiber characteristics. The results of this experiment suggest a significant trophic influence on the morphology and some contractile properties of skeletal muscle. Other mechanical and histochemical characteristics of rat soleus appear to be regulated by activity alone. Results of a portion of this work have been reported in preliminary form (Spector, 1984).

\section{Materials and Methods}

Contractile, morphometric, and histochemical parameters were assessed in soleus muscles of two groups of female Sprague-Dawley rats (170 to 190 $\mathrm{gm}$ ) after 2 or 1 weeks of DEN or paralysis with TTX applied to the motor nerve. One hindlimb of each animal of one group was denervated by excising 
a $10-\mathrm{mm}$ length of sciatic nerve midway between the thigh and knee. The contralateral limb was operated upon similarly, excluding nerve transection (control). In a second group of rats, an identical sciatic axotomy was performed. In the same region of the contralateral limb, electrical transmission was completely eliminated with TTX by modification of the perfusion technique by Betz et al. (1980; Spector, 1985). This produced an innervated but inactive muscle.

At the end of the experimental treatment, in vitro analyses of tension- and speed-related contractile properties of directly stimulated (curarized) soleus were performed. Soleus muscles were cleaned of connective tissue and tendon, blotted dry, weighed, and frozen for histochemical analyses. The details of materials and methods of treatment, data collection, and analysis are reported in detail in the preceding paper (Spector, 1985)

\section{Results}

Several control experiments were performed to verify that the TTX treatment did not result in partial DEN or changes in muscle properties attributable to direct effects of TTX (see Spector, 1985). In addition, only those animals which did not display a toe extension reflex throughout the period of TTX treatment, showed no in vivo electromyographic activity in musculature distal to the TTX nerve block, and proved to have complete block of action potentials to soleus upon sciatic nerve stimulation proximal to the cuff were included in the present results.

Morphology. Representative cross-sections of histochemically stained soleus muscles from rats treated for 4 weeks are shown in Figure 1. Compared to control fibers (Fig. 1a), fibers of denervated soleus ( $\Gamma \mathrm{ig} .1 \mathrm{C}$ ) were substantially reduced in size. Although TTXtreated muscle fibers (Fig. 1b) also appeared smaller compared to control fibers, all fibers of a given staining density of TTX-treated muscles were larger than those of contralaterally denervated muscle.

The distributions of alkaline myosin ATPase staining optical densities of muscle fibers were similar across muscles for a given experimental condition and time of treatment. Therefore, results for all muscles for each experimental treatment were pooled in Figure 2 to obtain the average percentage distribution of fibers of control, denervated, and TTX-treated muscles. After 2 (Fig. 2A) or 4 (Fig $2 B$ ) weeks, the population distribution of denervated fibers over lapped to a large degree that of TTX fibers. In addition, both twocell ( 0.65 density units demarcation value) and three-cell $(0.45$ and 0.75 density units demarcation values) $\chi^{2}$ tests did not reveal significant differences in frequency of fibers in any of the cells between TTX-treated and denervated muscles. Based on the demarcation value of 0.65 optical density units which divides normal soleus into slow twitch-oxidative (SO) and fast twitch-oxidativeglycolytic (FOG) subpopulations (Spector, 1985), two bimodally distributed subgroups remained after 2 or 4 weeks of treatment. Also, for a given fiber type, the mean staining optical densities were virtually identical for TTX and denervated fibers after 2 or 4 weeks. Consequently, although the percentage of SO fibers seen normally (Table I) was reduced significantly with TTX or DEN treatment, no differences in fiber type percentages were observed between denervated and TTX-treated muscles after 2 or 4 weeks of treatment (Table I).

Differences in muscle weights (MWs), whole muscle cross-sectional areas (M-CSAs), mean fiber cross-sectional areas (F-CSAs) and F-CSAs of SO and FOG fiber types between control, TTX, and denervated muscles are illustrated in Figure 3 . For all morphometric parameters of soleus studied after 2 or 4 weeks, significant differences were seen between control and TTX-treated or denervated muscles. Bilateral comparisons between TTX-treated and denervated muscles after either time of treatment revealed approximately $30 \%$ higher values for TTX-treated muscles in MW, M-CSA, and F-CSA. One exception was for the mean F-CSA of 2-week TTXtreated muscles, which was larger than that of denervated muscles by $20 \%$. Although detailed morphometric analyses of other affected hindlimb musculature were not performed in the present experiment, MWs of medial gastrocnemius (MG) and tibialis anterior (TA) were measured in three rats treated for 4 weeks. In each case, atrophy of TTX-treated muscles was substantially less than that seen in denervated muscles. TTX-disused MG and TA muscles were larger than contralateral muscles by $79.6 \%$ and $50.7 \%$, respectively (mean \pm SEM of MG, TTX: $262 \pm 14 \mathrm{gm}$; DEN: $146 \pm 11 \mathrm{gm}$; mean \pm SEM of TA, TTX: $201 \pm 24 \mathrm{gm}$; DEN: $134 \pm 4 \mathrm{gm}$ ).

Tension-related properties. The difficulties reported in maximally exciting chronically denervated (Drachman and Johnston, 1975; Finol et al., 1981) or TTX-disused (Spector, 1985) muscles were encountered in the present experiments, also. The majority of TTXtreated and denervated soleus muscles studied could not produce maximal twitch or tetanic $(50 \mathrm{~Hz}$ ) responses (see Fig. 7 of Spector, 1985) Nevertheless, for both TTX-treated and denervated muscles which did not produce maximal tension at lower voltages, a 50- to $60-\mathrm{V}$ square wave pulse lasting $0.5 \mathrm{msec}$ resulted in tension which appeared to be within about $90 \%$ of the maximal tetanic response without causing permanent mechanical damage.

Assuming nearly maximal activation with direct stimulation, it was found that the isometric twitch $\left(P_{t}\right)$ or tetanic tension $\left(P_{0}\right)$ was significantly imparied after 2 or 4 weeks of DEN or TTX disuse (Table II). The reductions in twitch tension produced by these muscles were not as great as the decreases in tetanic tension. In addition, significant reductions in specific tension in denervated and TTX-treated soleus were found at the end of 2 or 4 weeks of treatment, whether expressed as tetanic tension per weight, M-CSA, or F-CSA. When denervated and TTX-treated muscles were compared after 2 or 4 weeks of treatment, no differences were found in twitch tension. After both periods of treatment, however, maximal tetanic tension was significantly less in denervated soleus, resulting in a relatively enhanced twitch-to-tetanus ratio. The ability to generate tension relative to the degree of atrophy was similar after 2 weeks of DEN or TTX inactivation, whether expressed relative to MW, M-CSA, or F-CSA. After 4 weeks, though, less tension was generated relative to the reductions in denervated muscle mass or size. Thus, the specific tension of denervated soleus was less than that of TTXdisused muscle.

Speed-related properties. Isometric and dynamic measures of contractile speed are listed in Table III. Twitch responses of denervated and TTX-treated muscles were rarely maximal, as it was not possible to pass sufficient current to excite the whole musclc maximally without causing irreversible damage (see above, and Spector, 1985). Typical examples of the dependence of TTX-treated muscle tension on the duration and intensity of stimulation are illustrated in the previous paper (see Fig. 7 of Spector, 1985). The mechanical behavior of dencrvated muscle was essentially identical. Thus, in the present analyses, all speed-related responses were elicited with near-maximal stimuli of $60 \mathrm{~V}$ lasting $0.5 \mathrm{msec}$. Compared with control muscles, time-to-peak twitch tension (TPT) or relaxation time to half-peak tension (Half-RT) of TTX-inactivated muscle was not affccted after 2 or 4 weeks. In contrast, TPT and Half-RT of denervated soleus were significantly longer than values of either TTX-treated or control muscles after both times of treatment. The maximum rate of tension development $\left(d P_{o} / d t\right)$ of DEN- or TTXdisused soleus was reduced significantly from control after 2 or 4 weeks. Noverthclcss, the rate of tension development for 2 and 4 weeks was higher when expressed relative to maximally generated tension at $50 \mathrm{~Hz}\left(d P_{o} / d t: P_{o}\right)$. There were no differences in $d P_{o} / d t: P_{o}$ at either time between DEN and TTX treatments.

After 2 weeks of treatment, no differences were found between control and TTX-treated or denervated soleus in their absolute velocities of unloaded shortening $\left(V_{u s}\right)$. After 4 weeks of treatment, $V_{u s}$ of both TTX-treated and denervated muscles was higher than in the control soleus. However, after this period, there were no differences in $V_{u s}$ between TTX-treated and denervated muscles. The length at which maximal twitch tension was produced was not different across experimental conditions, suggesting that these $V_{u s}$ values were not influenced by gross geometrical differences in these muscles. 

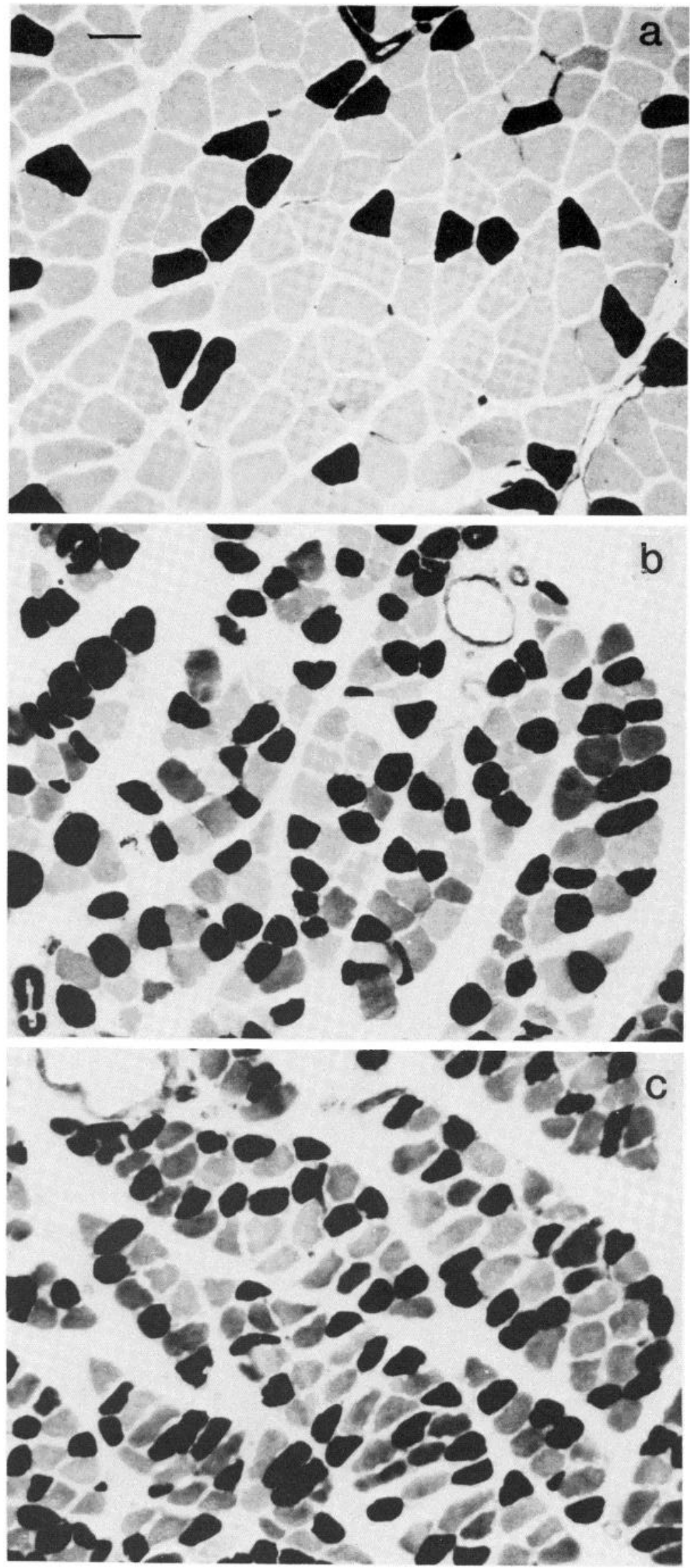

Figure 1. Photomicrographs of cross-sections of control (a), TTX (b), and denervated (c) muscles stained for the myofibrillar ATPase reaction, preincubated at $\mathrm{pH} 9.8$, after 4 weeks of treatment. Control soleus was obtained from unoperated limbs of a second group of weight-matched animals in which contralateral limbs were denervated. The calibration bar in a (upper left) equals $50 \mu \mathrm{m}$. 

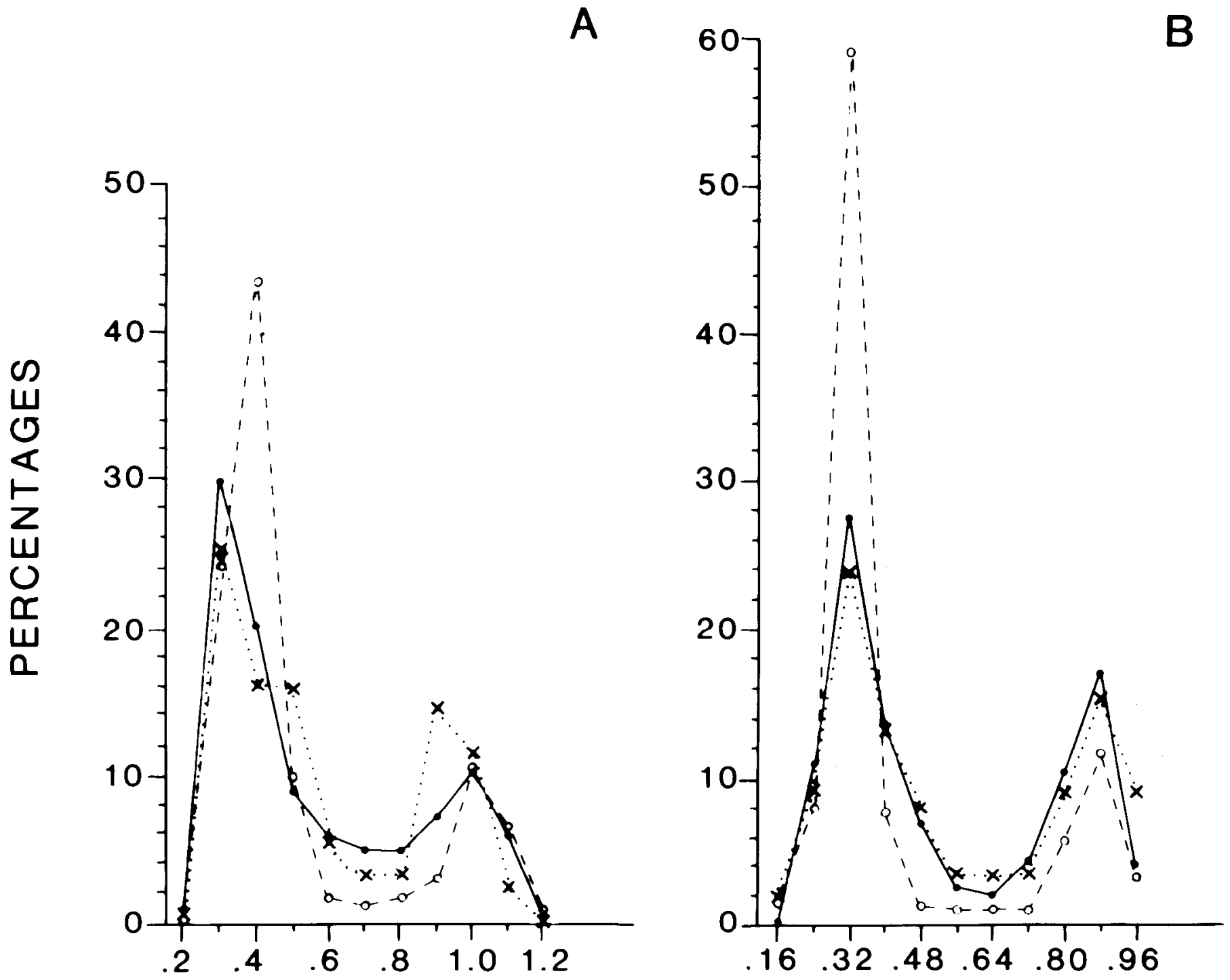

\section{ATPASE STAINING OPTICAL DENSITY}

Figure 2. Distribution of staining intensities of fibers of control $(---)$, TTX $(-)$, and denervated $(\ldots)$ muscles after $2(A)$ or $4(B)$ weeks of treatment. Fiber type distributions for individual muscles in each experimental treatment were similar and, therefore, were combined to produce this figure. The numbers of animals per condition and time are the same as in Figure 3. A demarcation value of 0.65 optical density units (see Spector, 1985) was used to delineate two discrete subpopulations of fibers (SO and FOG) for control, TTX, and denervated muscles.

TABLE I

Percentage of SO fibers in rat soleus after 2 or 4 weeks of denervation or $\pi \mathrm{X}$ inactivation

\begin{tabular}{ccc}
\hline Treatment & Two Weeks & Four Weeks \\
\hline Sham & $79.5^{a}$ & 78.3 \\
TTX & 65.8 \\
DEN & $64.5]$ & 61.8 \\
\hline
\end{tabular}

${ }^{a}$ Values are means \pm SEM. Sample sizes are the same as in Figure 3. Absence of brackets indicates significant difference between conditions.

\section{Discussion}

Previous experiments (Lømo and Rosenthal, 1972; Lømo et al., 1974; Purves and Sakmann, 1974) have suggested that many properties of muscle are regulated by neuromuscular activity, as transformations in morphological, electrophysiological, and contrac- tile characteristics of denervated muscle may be influenced with imposed electrical stimulation. Nevertheless, muscle mass and other physiological parameters are not restored to completely normal levels even after extended periods of stimulation ( $L \varnothing m o$ and Rosenthal, 1972; Lømo et al., 1974, 1980). One explanation for the incompleteness of the reversals could be the technical problems associated with artificial stimulation, including fiber damage due to electrode implantation, muscular contractions against uncontrolled loads, and the inability to mimic accurately natural motoneuronal firing patterns. Other studies, however, support the possibility of trophic interactions between nerve and muscle that are not replaced with stimulation (Harris, 1974; McArdle, 1983). Thus, by completely removing activity in an innervated neuromuscular system contralateral to denervation, the present experiment attempted to ascertain the existence of a trophic influence on contractile and histochemical properties of rat soleus muscle. 


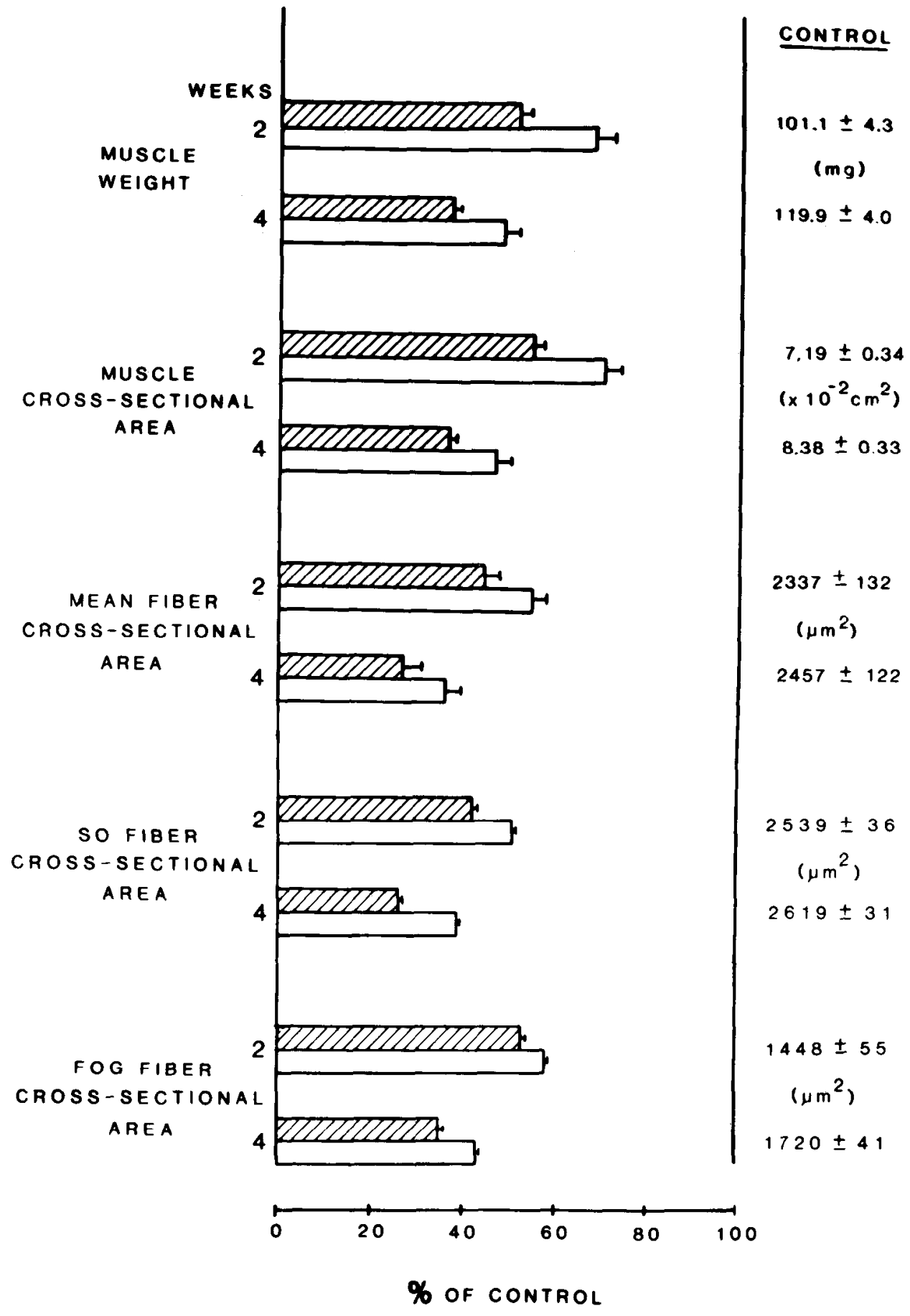

Figure 3. Muscle weight, whole muscle cross-sectional area, mean fiber cross-sectional area, and cross-sectional area of SO and FOG fiber types of TTX (open bars) and UEN (stippled bars) atter $2(n=8)$ or 4 $(n=6)$ weeks of treatment, expressed as percentages (mean \pm SEM) of control values ( 2 weeks, $n=6$; 4 weeks, $n=8$ ). Absolute values for control are listed to the right of the bars.

TABLE ॥

Tension properties of rat soleus after 2 or 4 weeks of denervation or $T X$ inactivation

\begin{tabular}{|c|c|c|c|c|c|c|c|}
\hline Trealment & $P_{1}(\mathrm{gm})$ & $P_{0}(\mathrm{gm})$ & $P t / P_{o}$ & $\mathrm{~m} / \mathrm{gm})$ & $P_{0} /$ & $\mathrm{CSA}\left(\mathrm{kg} / \mathrm{cm}^{2}\right)$ & $P_{0} / F-C S A\left(\mathrm{~kg} / \mathrm{cm}^{2}\right)$ \\
\hline \multicolumn{8}{|c|}{ Two weeks } \\
\hline Sham & $29.0 \pm 2.3^{2}$ & $132.0 \pm 9.3$ & $0.22 \pm 0.01$ & $1288 \pm$ & & $1.83 \pm 0.08$ & $1.90 \pm 0.10$ \\
\hline TTX & $15.3 \pm 1 . .7$ & $39.5 \pm 1.6$ & $0.39 \pm 0.01$ & $593 \pm$ & 27 & $0.81 \pm 0.037$ & $1.05 \pm 0.027$ \\
\hline \multicolumn{8}{|c|}{ Four weeks } \\
\hline Sham & $29.0 \pm 1.4$ & $144.7 \pm 6.8$ & $0.20 \pm 0.01$ & $1232 \pm$ & & $1.81 \pm 0.06$ & $1.82 \pm 0.06$ \\
\hline $11 x$ & $12.6 \pm 1.7$ & $32.7 \pm 3.6$ & $0.38 \pm 0.03$ & $575 \pm$ & & $0.85 \pm 0.04$ & $1.16 \pm 0.08$ \\
\hline
\end{tabular}

\footnotetext{
${ }^{a}$ Values are means \pm SEM. Sample sizesare the same as in Figure 3. Absence of brackets indicates significant difference between conditions.
} 
TABLE III

Speed properties of rat soleus after 2 or 4 weeks of denervation or $T X$ inactivation

\begin{tabular}{|c|c|c|c|c|c|}
\hline Treatment & $\begin{array}{c}\text { TPT } \\
(\mathrm{msec})\end{array}$ & $\begin{array}{l}\text { Half-RT } \\
(\mathrm{msec})\end{array}$ & $\begin{array}{c}d P_{o} / d t \\
(\mathrm{gm} / \mathrm{msec})\end{array}$ & $\begin{array}{c}d P_{o} / d t: P_{o} \\
(\%)\end{array}$ & $\begin{array}{c}V_{u s} \\
(\mathrm{~mm} / \mathrm{sec})\end{array}$ \\
\hline \multicolumn{6}{|l|}{ Two weeks } \\
\hline Sham & $89 \pm 5^{a} 7$ & $145 \pm 167$ & $839 \pm 107$ & $6.4 \pm 0.6$ & $56.1 \pm 10.8$ \\
\hline DEN & $111 \pm 5$ & $191 \pm 16$ & $298 \pm 22]$ & $8.7 \pm 0.3]$ & $61.8 \pm 6.5$ \\
\hline \multicolumn{6}{|l|}{ Four weeks } \\
\hline Sham & $85 \pm 37$ & $129 \pm 8$ & $859 \pm 21$ & $5.8 \pm 0.3$ & $55.4 \pm 0.3$ \\
\hline
\end{tabular}

${ }^{a}$ Values are means \pm SEM. Sample sizes are the same as in Figure 3 , except where otherwise noted $\left({ }^{b} n=4\right)$. Absence of brackets indicates significant difference between conditions.

Morphology. The extreme fluctuations in skeletal muscle mass due to such perturbations as high resistance weight training (Edgerton, 1976), joint immobilization (Solandt et al., 1943; Spector et al., 1982), or spinal cord transection (Johnson et al., 1982; Mayer et al., 1984) are attributed to changes in the pattern (Salmons and Sreter, 1976) or degree (Sreter et al., 1982) of neuromuscular activily. The finding of substantial muscular wasting as a result of virtually complete paralysis lasting 2 or 4 weeks is in accord with a significant role of some factor(s) associated with neuromuscular activity in regulating the mass of rat soleus muscle. The stimulus of active or passive lengthening has also been shown (Goldspink, 1978; Spector et al., 1982) to influence dramatically both MW and F-CSA. Nevertheless, the present results also demonstrate an additional factor that appears to influence muscle mass. In the case of 2 or 4 weeks of TTX paralysis, the intact motoneuron, although unable to propagate action potentials to the neuromuscular junction, exerts a significant trophic influence on both wet MW and fiber size of soleus. In fact, the observed differences in morphometric indices between DEN and neuromuscular paralysis may be underestimated if this trophic effect is in any way coupled to neurally evoked activity.

Tension-related properties. Similar bilatcral comparisons in the present experiment showed that, although 4 weeks of TTX treatment resulted in less tetanic tension than in control soleus, these disused muscles were relatively spared when compared with denervated muscles. When differences in mass or F-CSA between denervated and TTX-treated muscles were considered after 2 weeks of treatment, decreases in specific tension were equivalent between these two conditions. However, by 4 weeks of DEN, the further decrease in tetanic tension of denervated soleus was not paralleled by the atrophic response. Therefore, although specific tension of TTXtreated muscle was maintained at similar levels after 2 or 4 weeks of treatment, the specific tension of denervated soleus was reduced further with time. Similar progressive reductions in specific tension with time of DEN have been reported (Lømo, 1976; Finol et al., 1981).

The effects of DEN on electrophysiological characteristics of slow and fast twitch muscle include resting membrane depolarization, increased threshold for action potential production, reduced sodium gradient, increased membrane resistance and capacitance, and enhanced extrajunctional acetylcholine sensitivity (Hartzell and Fambrough, 1972; Linden and Fambrough, 1979; McArdle et al., 1980). These changes, in association with enhanced connective tissue content (Garcia-Bunuel and Garcia-Bunuel, 1980) reduce the excitability of denervated muscle (Lewis, 1972; Kean et al., 19/4; Drachman and Johnston, 1975) and have been attributed, in part, to the absence of a trophic stimulus (Harris, 1974; McArdle, 1983). Although the electrotonic properties of TTX-treated muscles have not been studied, the magnitude of changes in resting membrane potential or extrajunctional acetyicholine sensitivity of IIX-inactivated muscle is less than that observed in denervated muscle (Pestronk et al., 1976; Bray et al., 1979; Brown et al., 1982). Assuming that the electrotonic properties of TTX-inactivated muscies are also less affected than in denervated muscle, then the more greatly diminished excitability of denervated compared to TTX-disused muscle would be expected to result in diminished capacity to generate tension relative to muscle size, as found after 4 weeks of treatment.

Thus, the maintenance of specific tension in TTX-treated muscles could be accounted for by partial trophic regulation of electrophysiological properties of the cell membrane of TTX-disused muscle after 4 weeks of disuse. In addition, it is possible that the difference in specific tension between denervated and TTX-treated soleus by 4 weeks was due to a trophic influence on processes responsible for maintaining contractile protein concentrations relative to other cytoplasmic constituents.

Speed-related properties. Previous studies have reported conflicting results with respect to changes in TPT or Half-RT of slow twitch muscle. Although TPT is shorter in rabbit after 3 days of DEN, the twitch time course is prolonged in rat or cat soleus after 3 or 7 days, respectively, after axotomy (Syrovy et al., 1972; Drachman and Johnston, 1975; Finol et al., 1981). In addition to the prolongation of isometric twitch times of cat or rat soleus, a significant reduction in the maximal velocity of shortening of cat soleus has been reported (Kean et al., 1974).

In support of these earlier investigations of rat soleus, the results of the present study show that both TPT and Half-RT of denervated soleus are prolonged significantly compared with sham or TTX. treated muscles after 2 or 4 weeks of treatment. However, it was not possible in the majority of muscles to obtain a maximal twitch response. In fact, for a given magnitude of voltage passed across the muscle bath electrodes, increasing the pulse width from 0.1 msec to $0.9 \mathrm{msec}$ prolonged TPT by 10 to $15 \mathrm{msec}$. Also, comparing the responses elicited with a 40-V/0.1-msec pulse and a $100-\mathrm{V} / 0.9-$ msec pulse indicated prolongation of TPT by about $30 \mathrm{mscc}$. It is more likely that significant changes in electrophysiological properties of the muscle cell, including smaller action potentials of longer duration and reduced conduction velocities (Lewis, 1972), resulting in diminished ability to excite maximally all of the muscle's fibers (Lewis, 1972; Kean et al., 1974; Drachman and Johnston, 1975; Finol et al., 1981), were responsible for the variability of the twitch response obtained in denervated muscles. Therefore, the changes in twitch time parameters of denervated muscle may not be related necessarily to alterations in the underlying biochemical process of activation or to qualitative alterations in contractile proteins.

Strikingly similar difficulties were seen in attempting to maximally excite TTX-disused muscle, as well (see Fig. 9 of Spector, 1985). Thus, the significant differences in TPT between TTX-treated and denervated muscles cannot be attributed, at this time, to the presence or absence of a trophic influence. Rather, these differences may be due simply to the inability to maximally activate these whole muscles with voltages comparable to those used to maximally excite control or unoperated muscles, and show that determination of parameters related to the time course of the twitch are of little value in assessing the time course of changes in contractile activation or proteins which may occur under these experimental conditions. 
Increases in the isometric tetanic and dynamic speed-related properties after 4 weeks of TTX treatment are in accord with findings for another group of TTX-inactivated muscles (see Spector, 1985). However, the increases found in those same paramctcrs for denervated muscles are in direct opposition to the reduced maximal velocity of shortening of cat soleus and unaltered or reduced maximal rate of rise of isometric tetanic tension in cat or rat soleus (Kean et al., 1974; Drachman and Johnston, 1975; Finol et al., 1981). After 4 weeks of treatment in the present study, all TTX-treated or denervated muscles produced higher rates of tension development expressed relative to maximal tensions than did the control muscles. Although a portion of this enhanced rate of tension development could be explained by less than maximal tension development by TTX-treated or denervated muscles, correcting $d P_{v} / d t: P_{u}$ by as much as $20 \%$ would still yield substantially higher values than normal at 4 weeks. Significant differences in series elasticity, which may be estimated from the velocity experiments (Edman, 1979; Spector, 1985), between control, TTX-treated, and denervated muscles were not seen and, therefore, do not provide a likely source for these differences. Therefore, the provisional explanation for the enhanced $d P_{o} / d t: P_{o}$ in denervated or TTX muscle derives from changes in the biochemical processes of the contractile proteins.

Additional evidence for this hypothesis comes from determinations of $V_{u s}$ of TTX and denervated muscles. The $V_{u s}$ is believed to most accurately reflect the maximal rate at which cross-bridges can cycle. The finding of enhanced $V_{u s}$ suggests significant alterations in one or more processes related to activation and cycling of the contractile proteins. In fact, the values presented in Table III for denervated and TTX muscles may be underestimations of the true $V_{u s .}$ It has been suggested that the maximal velocity of shortening is dependent upon the level of activation of contractile proteins, as reducing the concentration of calcium exposed to the actin and myosin filaments in skinned fiber preparations produces diminished values of maximal shortening velocity (Julian and Moss, 1981). The reduced excitability of denervated or TTX-treated muscle fibers (see discussion above), then, might lead to a submaximal activation of contractile proteins, resulting in an underestimate of $V_{u s}$. I lowever, it must be noted that the dependence of shortening velocity upon calcium concentration has not been confirmed in other single muscle fiber preparations (Podolsky and Teichholz, 1970; Edman, 1979).

Histochemical observations of denervated and TTX-treated muscles are in direct accord with the contractile results. Significant increases in the number of FOG fibers were found in both TTXtreated and denervated muscles after 2 or 4 weeks of disuse. Similar fiber type conversions have been reported for denervated slow twitch muscle (Karpati and Engel, 1968; Nwoye et al., 1982) and slow muscles undergoing various degrees of reduccd activity (Booth and Kelso, 1973; Johnson et al., 1982; Musacchia et al., 1983; Roy et al., 1984). In addition, biochemically determined myosin ATPase activity and the relative proportion of fast light chains are significantly increased in rat soleus after chronic denervation (Nwoye et al., 1982). Therefore, based on the available histochemical, biochemical, and mechanical evidence, it appears that, at least for the rat soleus muscle, both chronic DEN and TTX inactivation lasting 4 weeks cause qualitative changes in one or more molecular processes involved in contractile protein activation. In addition, the lack of significant differences between TTX-treated and denervated soleus after 2 or 4 weeks with respect to histochemical or speed-related mechanical properties supports the hypothesis that these changes were brought about by the elimination of neuromuscular activity.

\section{References}

Bagust, J., D. M. Lewis, and R. A. Westerman (1981) Motor units in crossreinnervated fast and slow twitch muscle of the cat. J. Physiol. (Lond.) 314: 223-235

Betz, W. J., J. H. Caldwell, and R. R. Ribchester (1980) Sprouting of active nerve terminals in partially inactive muscles of the rat. J. Physiol. (Lond.) 303: $281-297$.
Booth, F. W., and J. R. Kelso (1973) Effect of hind-limb immobilization on contractile and histochemical properties of skeletal muscles. Pflugers Arch. 342: $231-238$.

Bray, J. J., J. I. Hubbard, and R. G. Mills (1979) The trophic influence of tetrodotoxin-inactive nerves on normal and reinnervated rat skeletal muscles. J. Physiol. (Lond.) 297: 479-491.

Brown, M. C., W. G. Hopkins, and R. J. Kcynes (1982) Comparison of effects of denervation and botulinum toxin paralysis on muscle properties in mice. J. Physiol. (Lond.) 327: 29-37.

Buller, A. J., and R. Pope (1977) Plasticity in mammalian skeletal muscle Philos. Trans. R. Soc. Lond. (Biol.) 278: 295-305.

Buller, A. J., J. C. Eccles, and R. M. Eccles (1960) Interactions between motoneurones and muscles in respect to the characteristic speeds of their responses. J. Physiol. (Lond.) 150: 417-439.

Buller, A. J., W. F. H. M. Mommaerts, and K. Seraydarian (1969) Enzymic properties of myosin in fast and slow twitch muscles of the cat following cross-reinnervation. J. Physiol. (Lond.) 205: 581-597.

Chan, M. K., V. R. Edgerton, G. E. Cosiow, Jr., H. Kurata, S. A. Rasmussen, and S. A. Spector (1982) Histochemical and physiological properties of cat motor units after self- and cross-reinnervation. J. Physiol. (Lond.) 332 : 343-361.

Close, R. (1969) Dynamic properties of fast and slow skeletal muscles of the rat after nerve cross-reunion. J. Physiol. (Lond.) 204: 331-346.

Drachman, D. B., and D. M. Johnston (1975) Neurotrophic regulation of dynamic properties of skeletal muscle: Effects of botulinum toxin and denervation. J. Physiol. (Lond.) 252: 657-667.

Edgerton, V. R. (1976) Neuromuscular adaptation to power and endurance work. Can. J. Appl. Sport Sci. 1: 49-58.

Edman, K. A. P. (1979) The velocity of unloaded shortening and its relation to sarcomere length and isometric force in vertebrate muscle fibres. J. Physiol. (Lond.) 291: 143-159.

Eisenberg, B. R., and S. Salmons (1981) The reoryariication of subcellular structure in muscle undergoing fast- to slow-type transformations. Cell Tissue Res. 220: 449-471.

Fernandez, H. L., and B. V. Ramirez (1974) Muscle fibrillation induced by blockade of axoplasmic transport in motor nerves. Brain Res. 79: 385395.

Finol, H. J., D. M. Lewis, and R. Owens (1981) The effects of denervation on contractile properties of rat skeletal muscle. J. Physiol. (Lond.) 319: $81-92$

Garcia-Bunuel, L., and V. M. Garcia-Bunuel (1980) Connective tissue metabolism in normal and atrophic skeletal muscle. J. Neurol. Sci. 47: 69-77.

Guldspirik, D. F. (1978) The influence of passive stretch on the growth and protein turnover of the denervated extensor digitorum longus muscle. Biochem. J. 174: 595-602.

Harris, A. J. (1974) Inductive functions of the central nervous system. Annu. Rev. Physiol. 36: 251-305.

Hartzell, H. C., and D. M. Fambrough (1972) Acetylcholine receptor distribution and extrajunctional density in rat diaphragm after denervation correlated with acetylcholine sensitivity. J. Gen. Physiol. 60: 248-262.

Hofmann, W. W., and J. H. Peacock (1973) Postjunctional changes induced by partial interruption of axoplasmic flow in motor nerves. Exp. Neurol. 41: 345-356.

Johnson, D. J., L. A. Sinith, E. Eldred, and V. R. Edgeton (1982) Exercise induced changes of biochemical, histochemical and contractile properties of muscle in cordotomized kittens. Exp. Neurol. 76: 414-427.

Julian, F. J., and R. L. Moss (1981) Effects of calcium and ionic strength on shortening velocity and tension development in frog skinned fibers. $J$. Physiol. (Lond.) 311: 179-199.

Kao, I., D. B. Drachman, and D. L. Price (1976) Botulinum toxin: Mechanism of presynaptic blockade. Science 193: 1257-1258.

Karpati, G., and W. K. Engel (1968) Correlative histochemical study of skcletal muscle after supraspinal denervation, peripheral nerve section and skeletal fixation. Neurology 18: 681-692.

Kean, C. J. C., D. M. Lewis, and J. D. McGarrick (1974) Dynamic properties of fast and slow twitch muscle of the cat. J. Physiol. (Lond.) 237: 103113.

Lavoie, P. -A., B. Collier, and A. Tennenhouse (1977) Role of skeletal muscle activity in the control of muscle acetylcholine sensitivity. Exp. Neurol. 54: $148-171$

Lewis, D. M. (1972) The effect of denervation on the mechanical and electrical properties of fast and slow mammalian twitch muscle. J. Physiol. (Lond.) 222: $51-75$. 
Linden, D. C., and D. M. Fambrough (1979) Biosynthesis and degradation of acetylcholine receptors in rat skeletal muscle: Effects of electrical stimulation. Neuroscience 4: 527-538.

Lømo, T. (1976) The role of activity in the control of membrane and contractile properties of skeletal muscle. In Motor Innervation of Muscle, pp. 289. 321. Academic Press, Inc., New York.

Lømo, T., and J. Rosenthal (1972) Control of Ach sensitivity by muscle activity in the rat. J. Physiol. (Lond.) 221: 493-513.

Lømo, T., R. H. Westgaard, and H. A. Dahl (1974) Contractile properties of muscle: Control by pattern of muscle activity in the rat. Proc. R. Soc. Lond. (Biol.) 187: 99-103.

Lømo, T., R. H. Westgaard, and L. Engebretsen (1980) Different stimulation patterns affect contractile properties of denervated rat soleus muscle. In Plasticity of Muscle, D. Pette, ed., pp. 297-309, Walter de Gruyter and Co., New York.

Mayer, R. F., R. E. Burke, J. Toop, B. Walmsley, and J. A. Hodgson (1984) The effect of spinal cord transection on motor units in cat medial gastrocnemius. Muscle Nerve 7: 23-31.

McArdle, J. J. (1983) Molecular aspects of the trophic influence of nerve on muscle. Prog. Neurobiol. 212: 135-198.

McArdle, J. J., L. Michelson, arid A. J. D'Alorizo (1980) Action potentials in fast- and slow-twitch mammalian muscles during re-innervation and development. J. Gen. Physiol. 75: 655-672.

Musacchia, X. J., J. M. Steffen, and D. R. Deavers (1983) Rat hind limb muscle responses to suspension hypokinesis/hypodynamia. Aviat. Space Environ. Med. 54: 1015-1020.

Nwoye, L., W. F. H. M. Mommaerts, D. R. Simpson, K. Seraydarian, and M. Marusich (1982) Evidence for a direct action of thyroid hormone in specifying muscle properties. Am. J. Physiol. 212: R401-R408.

Pestronk, A., D. B. Drachman, and J. W. Griffin (1976) Effects of muscle disuse on acetylcholine receptors. Nature 260: 252-253.

Pelte, D., M. E. Siritlr, H. W. Slaudle, and G. Vrbova (1973) Elfecis of lorigterm electrical stimulation on some contractile and metabolic characteris- tics of fast rabbit muscles. Pflugers Arch. 338: 257-272.

Pette, D., B. V. Ramirez, W. Muller, R. Simon, G. V. Exner, and R. Hildebrand (1975) influence of intermittent long-term stimulation on contractile, histochemical and metabolic properties of fibre populations in fast and slow rabbit muscles. Pflugers Arch. 361: 1-7.

Podolsky, R. J., and L. E. Teichholtz (1970) The relation between calcium ion concentration kinctics in skinned muscle fibrcs. J. Physiol. (Lond.) 211: $19-35$.

Purves, D., and B. Sakmann (1974) The effects of contractile activity on fibrillation and extrajunctional acetylcholine-sensitivity in rat muscle mlaintained in organ culture. J. Physiol. (Lond.) 237: 157-182.

Roy, R. R., R. D. Sacks, K. M. Baldwin, M. Short, and V. R. Edgerton (1984) Interrelationships of contraction time, $V_{\max }$, and myosin ATPase after spinal transsection. J. Appl. Physiol. 56: 1594-1601.

Salmons, S., and F. A. Sreter (1976) Significance of impulse activity in the transformation of skeletal muscle type. Nature 263: 30-34.

Solandt, D. Y., R. C. Partridge, and J. Hunter (1943) The effect of skeletal fixation on skeletal muscle. J. Neurophysiology 6: 17-22.

Spector, S. A. (1984) Regulation of muscle size by activity and non-activity related factors. Soc. Neurosci. Abstr. 10: 1060.

Spector, S. A. (1985) Effects of elimination of activity on contractile and histochemical properties of rat soleus muscle. J. Neurosci. 5: 000-000.

Spector, S. A., C. P. Simard, M. Fournier, E. Sternlicht, and V. R. Edgerton (1982) Architectural alterations of rat hind limb skeletal muscle immobilized at different lengths. Exp. Neurol. 76: 94-110.

Sreter, F. A., J. Gergely, S. Sallmons, and F. C. A. Romanul (1973) Synthesis by fast muscle of myosin light chains characteristic of slow muscle in response to long term stimulation. Nature 241: 17-19.

Sreter, F. A., K. Pinter, F. Jolesz, and K. Mabuchi (1982) Fast to slow transformalions of fast rnuscles in response to long-term phasic stimulation. Exp. Neurol. 75: 95-102.

Syrovy, I., E. Gutmann, and J. Melichna (1972) The effect of denervation on contraction and myosin properties of fast and slow rabbit and cat muscles. Physiol. Bohemoslov. 21: 353-359. 